\title{
Numerical Modelling of the Interaction Between Water Sprays and Hot Air Jets - Part I: Gas Phase Large Eddy Simulations
}

\author{
Tarek Beji, Georgios Maragkos, Setareh Ebrahimzadeh, Bart Merci \\ Department of Flow, Heat and Combustion Mechanics, Ghent University-UGent, B-9000, \\ Ghent, Belgium
}

\begin{abstract}
The paper reports a comprehensive set of large-eddy simulations (LES) of a turbulent hot air jet impinging onto a ceiling. The hot air source is a 72$\mathrm{mm}$ diameter circular nozzle with an exit temperature maintained at $205^{\circ} \mathrm{C}$. Three exit velocities have been tested: $3.3,4.2$ and $5.3 \mathrm{~m} / \mathrm{s}$, corresponding to Reynolds numbers of respectively 6800, 8600 and 10900 and Froude numbers of respectively $3.9,5.0$ and 6.3 . The horizontal aluminium ceiling plate of $1.22 \mathrm{~m}$ $\times 1.22 \mathrm{~m}$ has been placed at a distance of $590 \mathrm{~mm}$ above the hot air nozzle. This configuration has been examined experimentally by Zhou [Proceedings of the Combustion Institute, 2015] to characterize gas phase conditions prior to experiments which aim at studying the interaction between hot air jets and water sprays. This paper constitutes the first part of a numerical study that aims at assessing the current modelling capabilities of the two-phase flow configuration examined by Zhou [Proceedings of the Combustion Institute, 2015]. The results show that the centerline mean vertical velocity profiles of the vertical jet are predicted with maximum deviations of less than $6 \%$ from the experimental data at the condition of an appropriate set-up of the inflow conditions (i.e., geometry of the inlet and turbulence inflow boundary conditions). Furthermore, the best results were obtained with the dynamic Smagorinsky model for the turbulent viscosity. The modified Deardorff results are nevertheless very good given the substantial decrease in computational time (in comparison to the dynamic Smagorinsky model). A good prediction of the vertical jet allowed relatively
\end{abstract}

Preprint submitted to Fire Safety Journal

September 21, 2017 
good predictions of the ceiling jet maximum velocity, boundary layer thickness and Gaussian momentum width with maximum deviations of respectively $20 \%$, $1 \mathrm{~mm}$ and $18 \%$. The numerical modelling of the gas phase described in this paper can thus be relied upon in the two-phase simulations described in the companion paper [Part II: Two-phase flow simulations].

Keywords: Computational Fluid Dynamics (CFD), turbulence modelling, vertical jet, ceiling jet

\section{Introduction}

Water sprays are known to be an efficient means for fire control and suppression. In conjunction with experimental testing, modelling techniques are continuously being improved in order to be able to evaluate the performance of 5 water spray systems and their ability to create tenable conditions in the fire surroundings. Over the last decades, Computational Fluid Dynamics (CFD) has become a powerful technique that is not only used for academic and research purposes but also as a design tool in many areas of industry, including fire safety engineering. A continuous validation process is nevertheless required to ensure reliable CFD results. This is even more the case for complex two-phase flows characterized by a strong coupling between the gas phase (i.e., hot combustion products) and the liquid phase (i.e., water drops).

The configuration addressed in companion paper (i.e., Part II) consists of a ceiling-mounted water spray nozzle placed directly above the centre of a hot air jet issuing from a steel tube. The experimental campaign described in [1] aims primarily at providing a detailed and high quality experimental data for the purpose of assessing, improving and, eventually, validating the current CFD capabilities in the prediction of such two-phase flows. Experiments were first performed for a series of three hot air jets (corresponding to three injection 20 velocities) without a spray. Next, a water spray was characterized in terms of droplet size, velocity and water volume flow rate at two different distances from the nozzle (in the near-field and far-field of the spray) without hot air. Finally, 
the interaction of the three hot air plumes with the water spray is examined through combined gas-liquid velocity and droplet size measurements. Such a stepwise approach is suitable for CFD validation purposes in that it allows assessing first the gas phase and water spray modelling separately. If the level of agreement reached at the end of this stage is deemed high enough then, a potential disagreement between experimental data and numerical results for the spray-jet interaction could be explained by the need for improvement in submodels that directly act on the interaction between the two phases, such as the evaporation model.

In this paper (i.e., Part I), the focus is put on the gas phase simulations. In other words, we would like to make sure that the flow field from the hot air is well predicted because any deviation in the hot air momentum at any height will 35 directly impact the spray-plume interaction since the latter is mainly governed by the competition between the momentum of the plume and the momentum of the spray. In 2], numerical simulations of the experiments described above have been performed with the CFD code FireFOAM, using the Large Eddy Simulation (LES) approach. Results of the so called isolated thermal plumes were however limited to near-inlet velocity and temperature profiles. A more thorough numerical study, also performed with FireFOAM (with the LES approach), has been conducted in [3] where the focus was put on the influence of the modelling of the turbulent viscosity as well as the turbulence inflow boundary conditions. The obtained results were generally satisfactory. Nevertheless, we observed that the best results were obtained without any subgrid scale (SGS) modelling for a cell size of $4 \mathrm{~mm}$ that is not fine enough to have a fully resolved flow. This relatively surprising finding encouraged us to use the Fire Dynamics Simulator (another CFD package that is widely used in the fire safety community (4 [5]) in order to uncover potential differences in numerical dissipation between the two codes. Another point of interest in redoing the exercise with FDS is the treatment of turbulence inflow boundary conditions using the Synthetic Eddy Method (SEM), as opposed to the method of random spots relied upon in FireFOAM. 
The general objective of this paper and the companion paper remains though to deliver a complete, comprehensive and careful CFD analysis of the sprayplume interaction with FDS for validation purposes. The comparison with FireFOAM remains for now only at the level of observations of the differences between the two codes. A detailed comparative study (which is out of the scope of the current paper) requires more work and is certainly worth undertaking in 60 the future.

\section{Experimental set-up}

In [1] a vertical jet of hot air in a quiescent environment is examined. The hot air source is a $72 \mathrm{~mm}$-diameter $(D)$ circular nozzle issuing from a $254 \mathrm{~mm}$ long steel tube. The hot air exit temperature has been maintained at $T_{0}=205$

${ }_{65}{ }^{\circ} \mathrm{C}$ at $30 \mathrm{~mm}$ above the nozzle exit. Three exit velocities, $w_{0}$, have been tested: $3.3,4.2$ and $5.3 \mathrm{~m} / \mathrm{s}$. A $3 \mathrm{~mm}$-thick horizontal aluminium ceiling plate of 1.22 $\times 1.22 \mathrm{~m}$ has been placed at a distance of $H=590 \mathrm{~mm}$ above the hot air nozzle (see Fig.1).

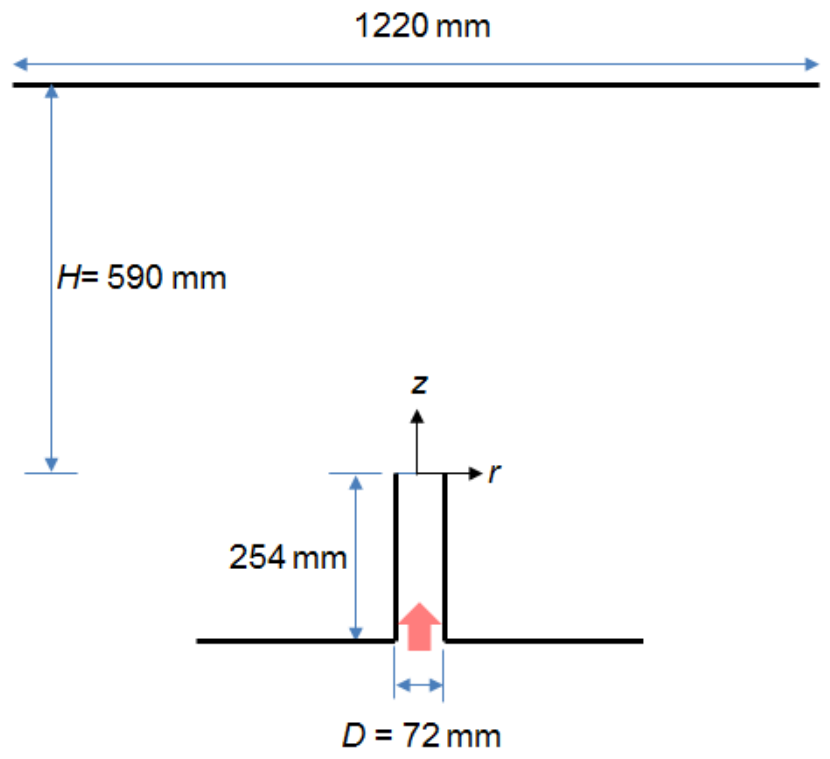

Figure 1: Schematic of the experimental set-up. 
Mean velocities (radial and vertical directions) and velocity fluctuations (vertical jet and the ceiling jet have been measured using the laser-based Particle Image Velocimetry (PIV) technique. Temperature measurements have not been performed, except at $30 \mathrm{~mm}$ above the nozzle exit. More details on the experimental set-up can be found in [1]. numbers calculated as:

$$
\begin{aligned}
& R e=\frac{w_{0} D}{\nu} \\
& F r=\frac{w_{0}}{\sqrt{g D}}
\end{aligned}
$$

where $g$ is the gravitational acceleration and $\nu$ is the kinematic viscosity of hot air (taken as $\nu=3.5 \times 10^{-5} \mathrm{~m}^{2} / \mathrm{s}$ ). The source is further characterized in [1] in terms of a densimetric Froude number calculated as:

$$
F r_{\rho}=\frac{w_{0}^{2} T_{a m b}}{g D\left(T_{0}-T_{a m b}\right)}
$$

where $T_{a m b}$ is the ambient temperature taken as $T_{a m b}=20^{\circ} \mathrm{C}$.

The values of $R e, F r$ and $F r_{\rho}$ are displayed in Table 1 for the three cases. It is noteworthy to mention that the obtained values of the Froude number are significantly higher than the ones typically encountered in fires. The obtained vertical jets must then be considered as momentum jets rather than buoyancydriven plumes. In the general context of fire suppression, this can be seen as a limitation of the current study because the Froude numbers are not representative of fire plumes. Nevertheless, the main purpose in 1 is to generate data for CFD validation rather than scaling up the results and deriving correlations for fire sources.

\section{Numerical modelling}

The Fire Dynamics Simulator is a CFD code, initially developed for lowMach number buoyancy-driven flows. However, the latest version has also been successfully applied to high-momentum flows (e.g., $u_{0}=7.2 \mathrm{~m} / \mathrm{s}$ and $R e=5100$ 
Table 1: Reynolds, Froude and densimetric Froude numbers for the three cases.

\begin{tabular}{|c|c|c|c|c|}
\hline & $w_{0}(\mathrm{~m} / \mathrm{s})$ & $R e$ & $F r$ & $F r_{\rho}$ \\
\hline Case 1 & 3.3 & 6789 & 3.9 & 24 \\
\hline Case 2 & 4.2 & 8640 & 5.0 & 40 \\
\hline Case 3 & 5.3 & 10903 & 6.3 & 63 \\
\hline
\end{tabular}

95 in 4, 5. The most relevant aspects for the case at hand are recalled here.

\subsection{Turbulent viscosity models}

Turbulence is modeled using the Large Eddy Simulation (LES) technique. Four models are available in [4, 5] for the modelling of the turbulent viscosity: the modified Deardorff (default model), the Smagorinsky (constant and dynamic) and the Vreman model. Two options have been tested in this work, namely the modified Deardorff and the dynamic Smagorinsky model. A third option consists of considering no sub-grid scale (SGS) modelling.

In the modified Deardorff model, the turbulent dynamic viscosity is expressed as:

$$
\mu_{t}=\bar{\rho} C_{v} \Delta \sqrt{k_{S G S}}
$$

where $C_{v}$ is a constant taken as $0.1, \Delta$ the filter width (taken as the cubic root of the cell volume) and $k_{S G S}$ is the subgrid scale kinetic energy taken from an algebraic relationship based on scale similarity. The calculation of $k_{S G S}$ in 4, 5] is different from the original paper of Deardorff where the kinetic energy, $k$, is calculated using a transport equation. That is why the term modified is added here.

In the Smagorinsky model, the turbulent dynamic viscosity is expressed as:

$$
\mu_{t}=\bar{\rho}\left(C_{s} \Delta\right)^{2}|\tilde{S}|
$$

where $C_{s}$ is the Smagorinsky constant calculated locally using the dynamic procedure and $|\tilde{S}|$ is the strain rate. Turning off the SGS modelling was performed 115 


\subsection{Turbulence inflow boundary conditions}

Turbulence inflow boundary conditions are modeled using the Synthetic Eddy Method (SEM) that has been developed in [7]. This method consists of creating a number, $N_{e d d y}$, of artificial eddies of a given size, $\ell$, which are in motion at the level of the inlet in order to generate synthetic turbulence that resembles more a real turbulent flow than obtained with a simple white noise. The SEM model allows creating more realistic turbulence inflow boundary conditions without having to model a full pipe to emulate a pipe flow as it is the case in the experiments at hand. The velocity fluctuation is expressed as:

$$
u_{i}^{\prime}=\frac{1}{\sqrt{N_{e d d y}}} \sum_{k=1}^{N_{e d d y}} a_{i j} \varepsilon_{j}^{k} f_{\ell_{i j}(x)}\left(x-x^{k}\right)
$$

where $a_{i j}$ is the Cholesky decomposition of the Reynolds stress tensor, $\varepsilon_{j}^{k}$ are independent random variables taken from any distribution with zero mean and unit variance, and $f$ is a shape function which provides the velocity distribution of the eddies located at $x^{k}$. The subscripts $i$ and $j$ denote respectively the velocity component and the spatial direction. Expression (6) allows the generation of non-isotropic velocity fluctuations at the inlet. If isotropic structures are produced, the three dimensional length scales, $\ell_{i j}$, defining the structure of turbulent eddies for each velocity component $i$ in each direction $j$, are replaced by one value, i.e., $\ell_{i j}=\ell$. In the case at hand, isotropic turbulence is prescribed at the inflow boundary condition.

\subsection{Velocity boundary condition at the ceiling}

A zero-gradient $\mathrm{BC}$ is used for the tangential velocity and the velocity divergence as an outflow $\mathrm{BC}$. The near-wall velocity is calculated using a wall function which is expressed as:

$$
\begin{gathered}
u^{+}=y^{+} \quad \text { for } \quad y^{+}<11.81 \\
u^{+}=\frac{1}{\kappa} \ln y^{+}+B \quad \text { for } \quad y^{+}>11.81
\end{gathered}
$$

where $y^{+}$and $u^{+}$are respectively the non-dimensional wall-normal distance and streamwise velocity, $\kappa=0.41$ is the von Karman constant and $B=5.2$. 
An alternative approach to the wall function, which has also been tested here, is the no-slip boundary condition which implies that the tangential gas velocity at a surface is zero, i.e., $u_{w}=0$.

\section{Simulation test cases}

\subsection{Vertical jet simulations}

Prior to the gas-phase simulations of the full domain, the focus was initially put on the vertical jet. The computational domain has been reduced, as shown in Fig. 2, in order to reduce the computational times. Several modelling options have been examined first with respect to essentially $(i)$ the geometrical configuration of the inlet, (ii) the turbulent viscosity model and (iii) the cell size.

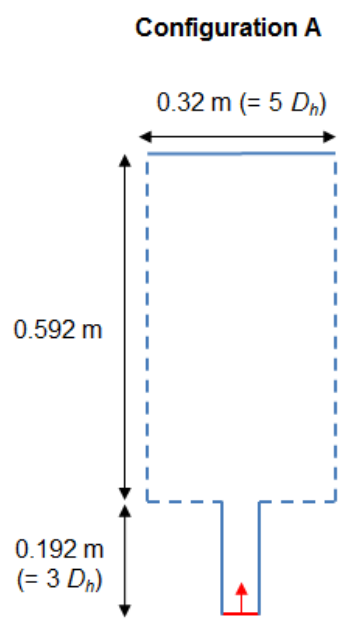

Configuration B

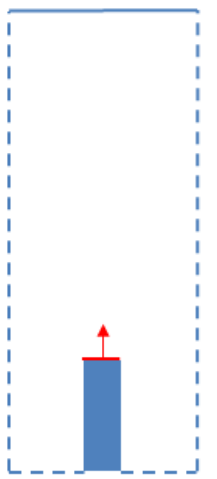

Configuration $\mathrm{C}$

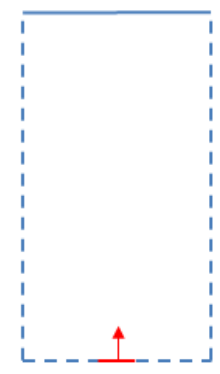

Figure 2: Configurations used for the simulation of the jet. The solid red line represents the inlet of hot air (as indicated by the arrow). The solid blue lines represent inert walls and ceiling. The dashed blue lines represent OPEN boundary conditions.

\subsubsection{Geometrical configuration of the inlet}

As shown in Fig. 2, three geometrical configurations were examined. In the

first one, i.e., configuration A, a pipe length of 3 hydraulic diameters $\left(3 D_{h}\right)$ is 
considered. This means that the inlet properties (mean velocity and temperature and turbulence inflow boundary conditions) are specified in configuration A, at $3 D_{h}$ below the inlet exit. Note that in most of the simulations performed herein, a square source is modeled with a length $L=D_{h}=0.064 \mathrm{~m}$ (which yields almost the same inlet area as in the experiments, with a deviation of about $0.6 \%$ ) because only Cartesian coordinates are used in FDS. Nevertheless, the influence of the circular shape has been carried out for one simulation by using the stair stepping method to mimic the round shape of the tube (i.e., pipe) with $L=D=0.072 \mathrm{~m}$. However, in this approach, the inlet area is 8.8 $\%$ lower than the actual one. The pipe walls and ceiling are modeled as INERT (see solid lines in Fig.2). The dashed lines in Fig. 2 represent OPEN boundary conditions. In configuration B, the pipe was replaced by a solid obstacle and the inlet conditions were specified at its top. In configuration $\mathrm{C}$, the computational domain has been reduced in height, restricted to the inlet to ceiling height. In this configuration the inlet is thus flush with the domain boundary.

\subsubsection{Turbulent viscosity model}

As mentioned above, three modelling options have been tested in this work regarding the turbulent viscosity: (i) dynamic Smagorinsky, (ii) modified Deardorff, and (iii) no SGS modelling.

Three cell sizes were tested: $2 \mathrm{~mm}, 4 \mathrm{~mm}$ and $8 \mathrm{~mm}$. The cell size for the base case is $4 \mathrm{~mm}$.

\subsubsection{Other simulation settings}

A uniform structured mesh was used with a cell size of $4 \mathrm{~mm}$ for most of the simulations. Furthermore, in configurations A, B and $\mathrm{C}$ the domain is divided respectively into 9,12 , and 8 blocks of meshes, each block of mesh assigned to one processor. A series calculation (i.e., with one processor) has also been performed for configuration A to check if there are any differences with parallel computing and to evaluate the potential gain in computational 

viscosity model, simulation time and number of processors.

Table 2: Vertical-jet simulation model settings.

\begin{tabular}{|l|c|c|}
\hline & Base case & Alternative \\
\hline Domain & configuration A & configuration B or C \\
\hline Inlet shape & square & circular \\
\hline Turbulence model & dynamic Smagorinsky & modified Deardorff or no SGS \\
\hline Cell size & $4 \mathrm{~mm}$ & $2 \mathrm{~mm}$ or $8 \mathrm{~mm}$ \\
\hline Simulation time & $30 \mathrm{~s}$ & $60 \mathrm{~s}$ \\
\hline Number of processors & 9 & 1 \\
\hline
\end{tabular}

Several preliminary simulations (not detailed here) using the base case settings in Table 2 have been performed to find the SEM model parameters that provide a good agreement with the centreline mean vertical velocity. These parameters are $N_{e d d y}=1000$ and $\ell=0.1 D_{h}=6.4 \mathrm{~mm}$. Furthermore, the inlet 195 mean velocities and fluctuations are displayed in Table 3.

Table 3: Inlet velocities and fluctuations.

\begin{tabular}{|c|c|c|c|c|}
\hline & Configuration & $w_{0}(\mathrm{~m} / \mathrm{s})$ & $w_{0}^{\prime}(\mathrm{m} / \mathrm{s})$ & $I_{w}=\left(w_{0} / w_{0}^{\prime}\right) \times 100(\%)$ \\
\hline Case 1 & $\mathrm{~A}$ & 3.10 & 0.41 & $13 \%$ \\
\hline Case 1 & $\mathrm{~B}$ & 3.30 & 0.18 & $5 \%$ \\
\hline Case 1 & $\mathrm{C}$ & 3.30 & 0.18 & $5 \%$ \\
\hline Case 2 & $\mathrm{~A}$ & 3.95 & 0.52 & $13 \%$ \\
\hline Case 3 & $\mathrm{~A}$ & 5.00 & 0.65 & $13 \%$ \\
\hline
\end{tabular}




\subsection{Ceiling-jet simulations}

Figure 3 shows the domain and boundary conditions used for the simulations of the ceiling jet. For the base case, the main domain was divided into 9 blocks of uniform and structured mesh with a cell size of $4 \mathrm{~mm}$. A $10^{\text {th }}$ block of mesh was allocated to the inlet pipe. Ten processors were used (1 processor for each block of mesh). Table 4 displays the model settings that were tested in the ceiling-jet configurations.

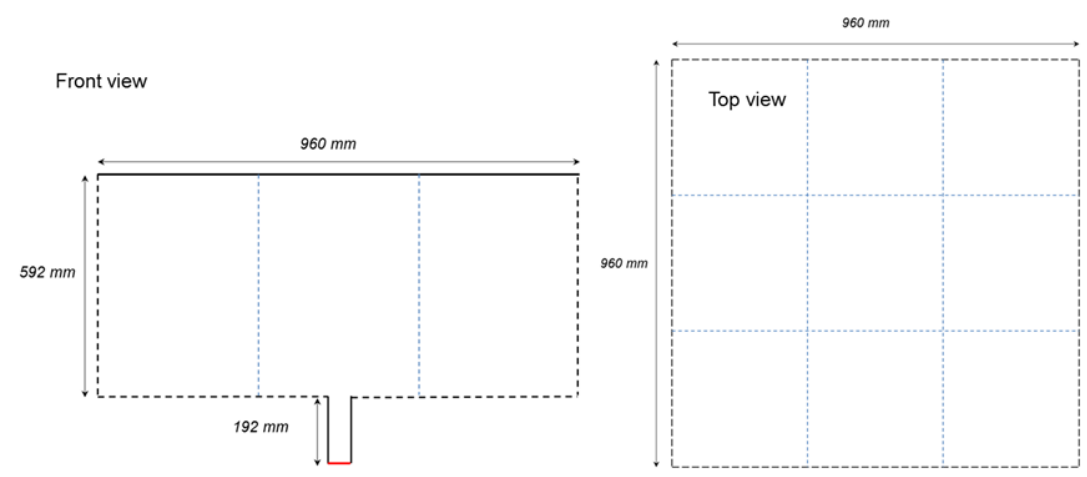

Figure 3: Computational domain for the ceiling jet calculations.

Table 4: Ceiling-jet simulation model settings.

\begin{tabular}{|l|c|c|}
\hline & Base case & Alternative \\
\hline Domain & $0.96 \mathrm{~m} \times 0.96 \mathrm{~m} \times 0.592 \mathrm{~m}$ & $1.28 \mathrm{~m} \times 1.28 \mathrm{~m} \times 0.592 \mathrm{~m}$ \\
\hline Turbulence model & dynamic Smagorinsky & modified Deardorff \\
\hline Velocity BC & wall function & no-slip \\
\hline Thermal BC & heat transfer & adiabatic \\
\hline Cell size & $4 \mathrm{~mm}$ & $2 \mathrm{~mm}$ \\
\hline
\end{tabular}




\section{Results}

\subsection{Vertical jet simulations}

Fig. 5, the velocity decreases unexpectedly near the inlet up to about $z=35$ $\mathrm{mm}$ before starting to rise (normally) due to the buoyancy-induced acceleration. Extending the domain to minimize the effect of the OPEN boundary condition, as shown in Fig. 2 for configuration B, led to a result similar to configuration

SEM currently implemented in FDS does not preserve the velocity divergence. 


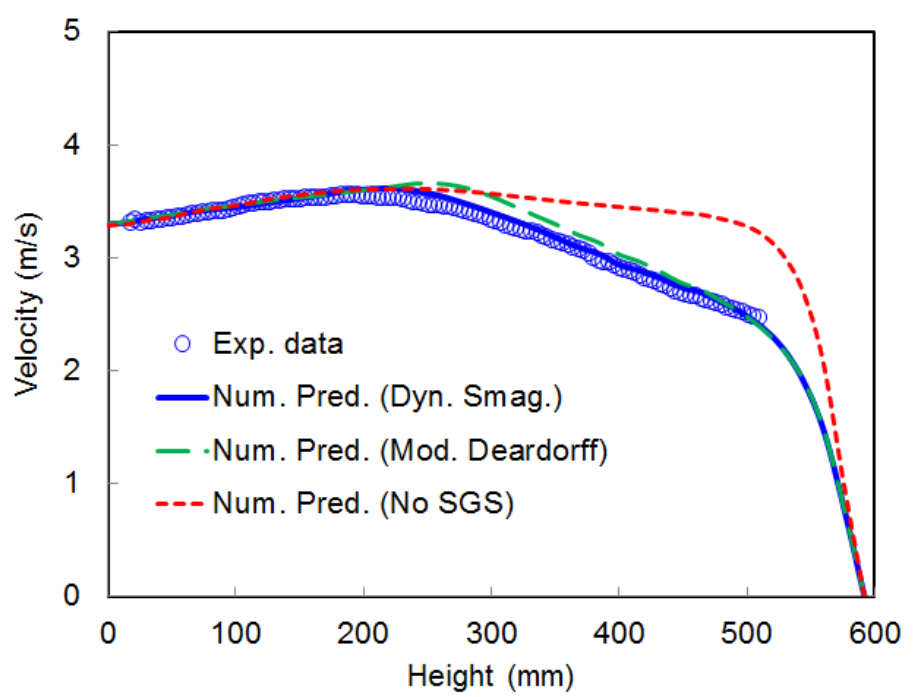

Figure 4: Influence of the turbulent viscosity model on the predictions of the centreline vertical velocity for case 1.

There is a divergence preserving variant of SEM by Poletto et al. 8 that will be implemented in a future version of FDS.

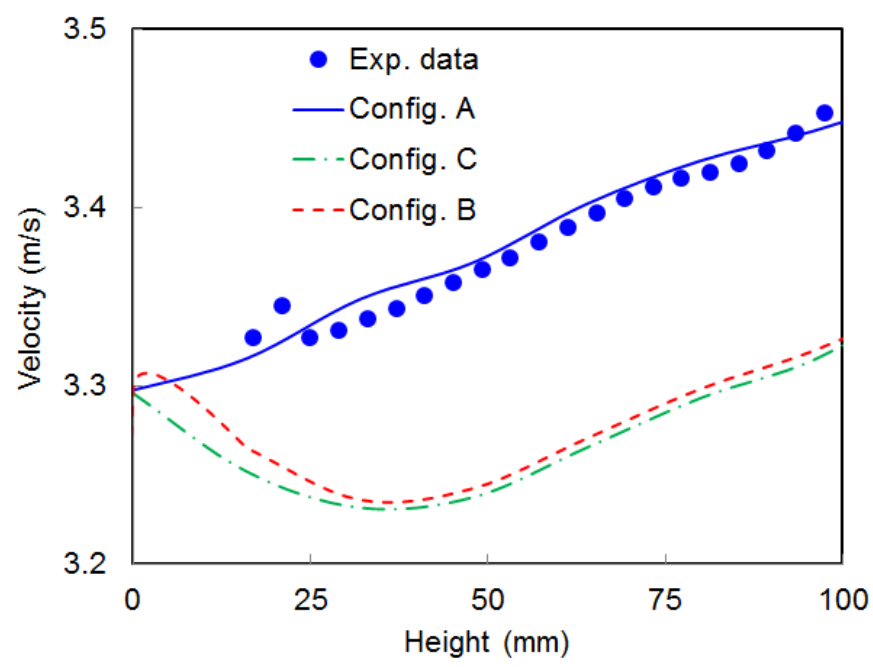

Figure 5: Influence of the inlet configuration on the predictions of the centreline vertical velocity near the inlet for case 1 . 


\subsubsection{Mesh sensitivity analysis (case 1)}

The results of the mesh sensitivity analysis are displayed in Figs. 6 to 8 . Cell sizes of $2 \mathrm{~mm}$ and $4 \mathrm{~mm}$ yield very similar results for the centreline and radial profiles of mean vertical velocity (see Figs. 6 and 7). There is however a slightly wider profile in the near-field at $z=1 D$. The differences in the vertical velocity fluctuations are more significant, especially in the near-field. Based on this mesh sensitivity analysis, a cell size of $4 \mathrm{~mm}$ is deemed appropriate for the case at hand.

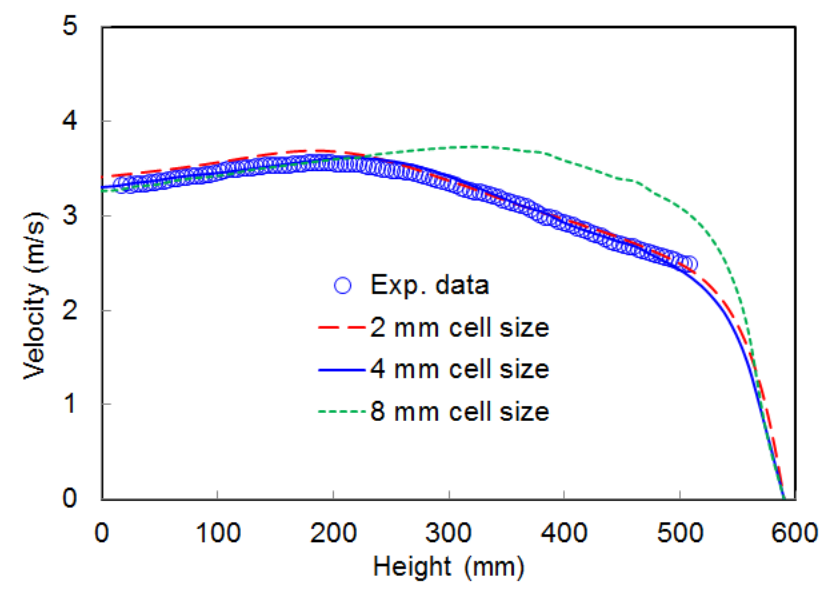

Figure 6: Influence of the cell size on the predictions of the centreline vertical velocity for case 1.
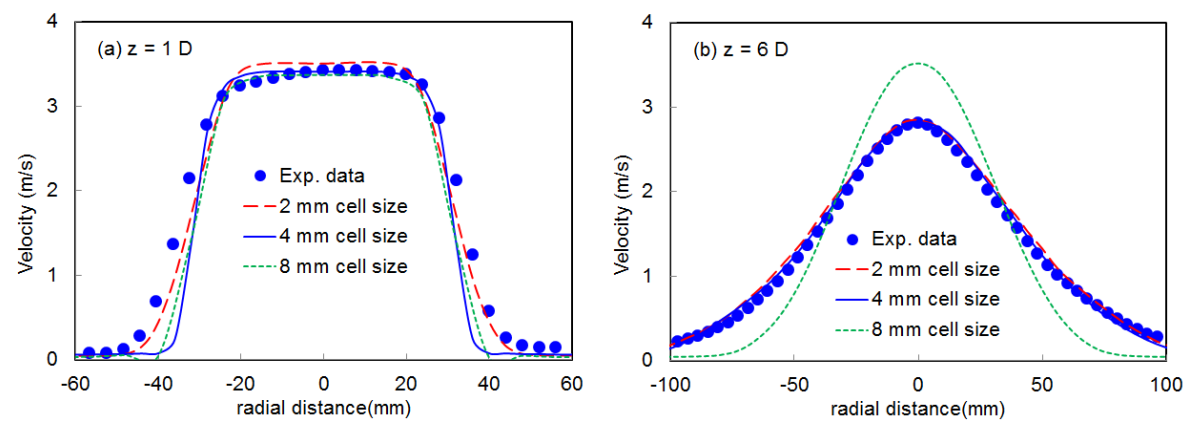

Figure 7: Influence of the cell size on the predictions of the radial profiles of the mean vertical velocity for case 1 at (a) $z=1 D$ and (b) $z=6 D$. 

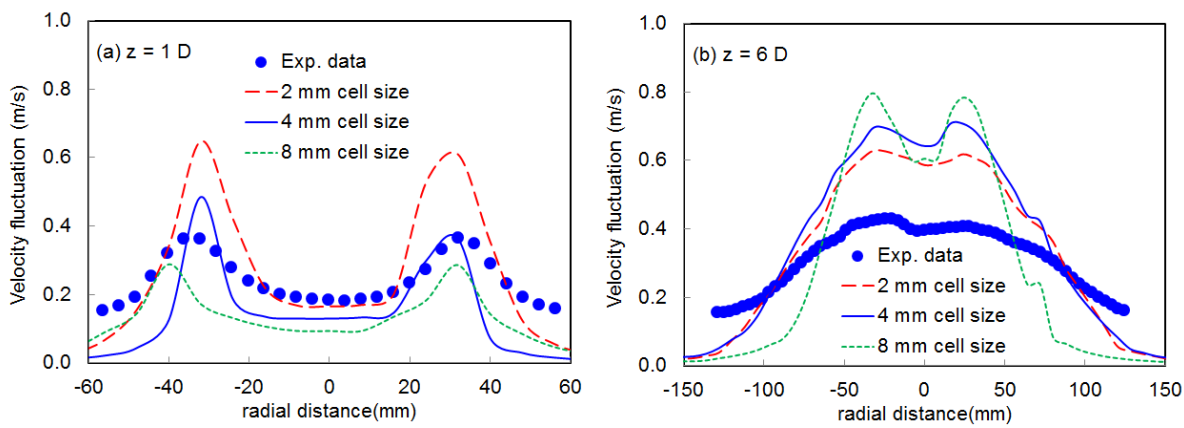

Figure 8: Influence of the cell size on the predictions of the radial profiles of the vertical velocity fluctuations (i.e., rms) for case 1 at (a) $z=1 D$ and (b) $z=6 D$.

\subsubsection{Influence of the shape of the inlet (case 1)}

Figure 9 shows that the approximated round configuration provides, as expected, a better agreement (with a wider profile) for the mean vertical velocity near the inlet (at $z=1 D$ ) because the inlet diameter length is the same as in the experiments, $L=D=0.072 \mathrm{~m}$ (as opposed to the square inlet with $L=D_{h}=0.064 \mathrm{~m}$ ). For the second-moment profiles (see Figs. 10 and 11), the round inlet configuration clearly outperforms the square inlet configuration, especially for the turbulent shear stress at $z=1 D$ (see Fig.11a). The differences further downstream, for example at $z=6 D$ (as shown in Figs. 10b and 11b), are rather marginal. Recalling the fact that the actual inlet area could not be recovered (8.8\% underestimation) with the round inlet, it is deemed best to keep the square inlet configuration for the remainder of the simulations.

\subsubsection{Main results for the three cases.}

Figure 12 shows the results for the centerline vertical velocity for all the three cases using the dynamic Smagorinsky model and configuration A. Agreement of simulation results with experimental data is very satisfactory. The radial profiles of mean vertical velocity for the three cases are displayed in Fig. 13. The predicted profiles are slightly narrower than the experimental ones at $z=1 D$. As explained earlier, this could be attributed to the cell size or the square shape of the inlet. 


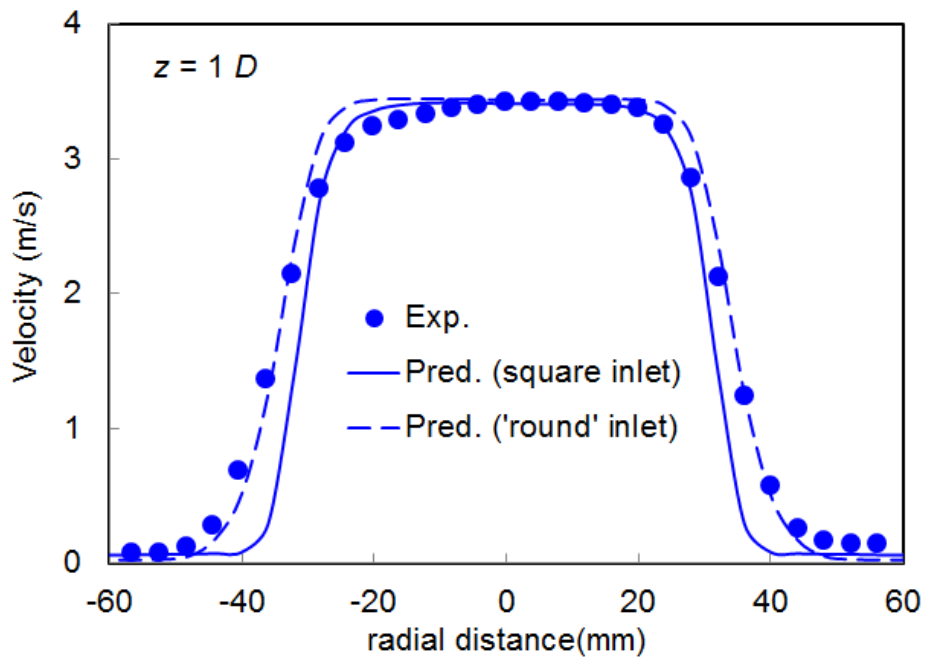

Figure 9: Influence of the inlet shape on the radial profile of mean vertical velocity for case 1 at $z=1 D$.
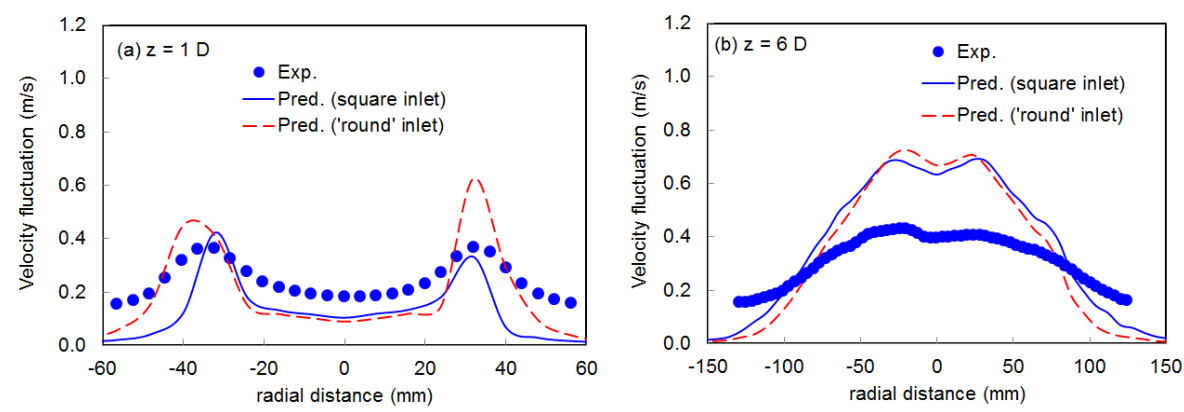

Figure 10: Influence of the inlet shape on the radial profiles of the vertical velocity fluctuations (i.e., rms) for case 1 at (a) $z=1 D$ and (b) $z=6 D$.

The results for the vertical velocity fluctuations and the turbulent shear stress for the three cases are displayed in respectively Figs. 14 and 15. Figure 14a shows that the vertical velocity fluctuations are relatively well predicted at ${ }_{265} z=1 D$, especially on the centerline for cases 2 and 3. Further downstream, at $z=6 D$, the vertical velocity fluctuations are significantly overpredicted. We note that, as opposed to the numerical profiles, the experimental vertical velocity fluctuations are similar for the three cases despite significant differences 

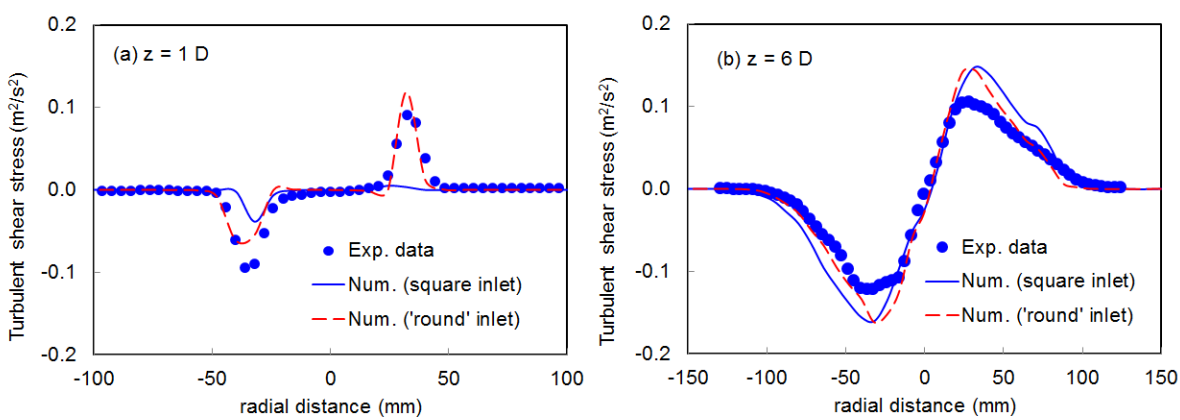

Figure 11: Influence of the inlet shape on the radial profiles of the turbulent shear stress (i.e., Reynolds stress) for case 1 at (a) $z=1 D$ and (b) $z=6 D$.

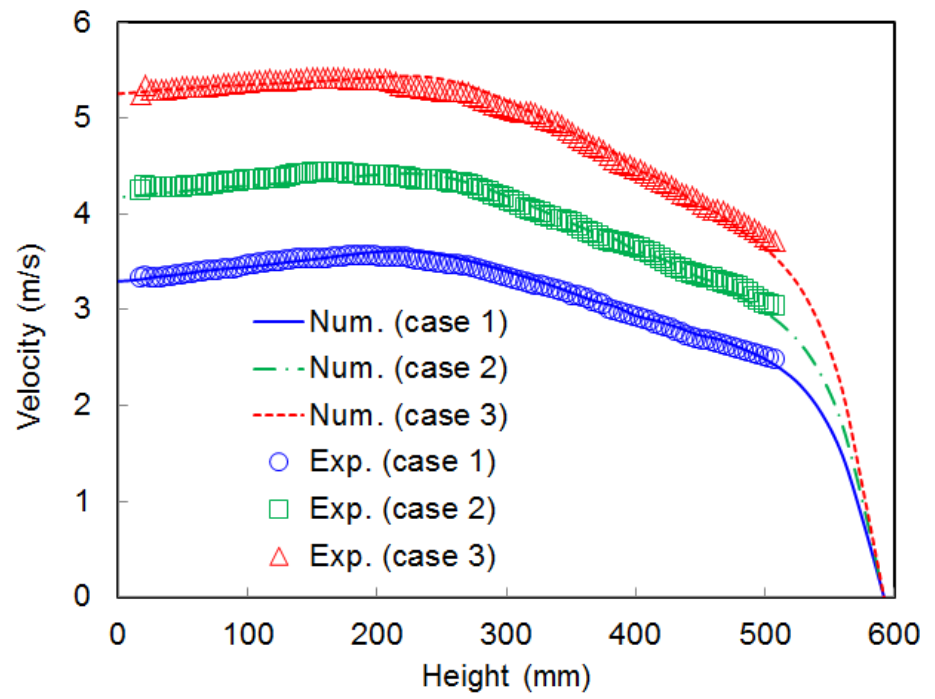

Figure 12: Predictions of the centreline mean vertical velocity profiles for cases 1, 2 and 3.

in the mean vertical velocity profiles, which is quite unexpected and could be attributed to experimental uncertainties.

The turbulent shear stresses are generally well predicted at $z=6 D$ and significantly underpredicted at $z=1 D$, which is attributed, as explained above, to the square shape of the inlet. 

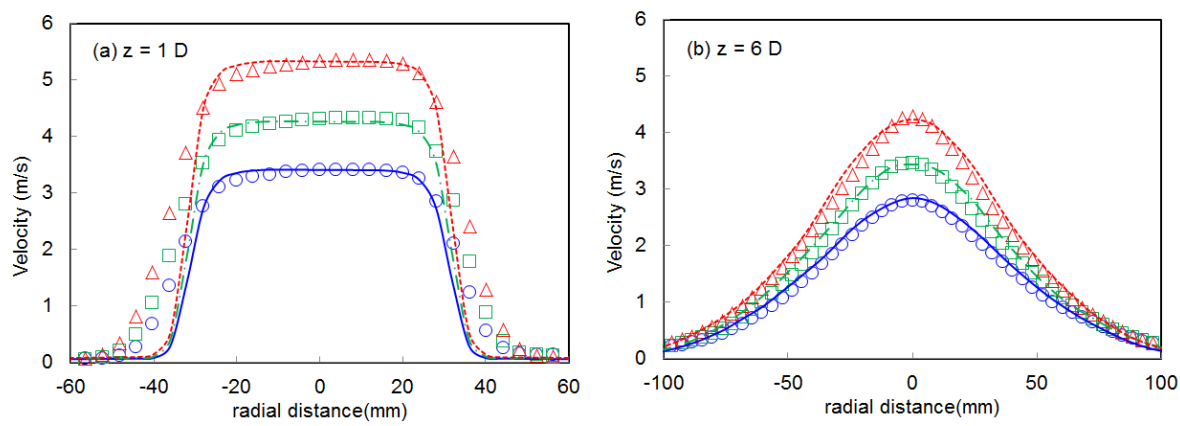

Figure 13: Predictions of the radial mean vertical velocity profiles for cases 1, 2 and 3 at (a) $z=1 D$ and (b) $z=6 D$. (see Fig. 12 for the legend)
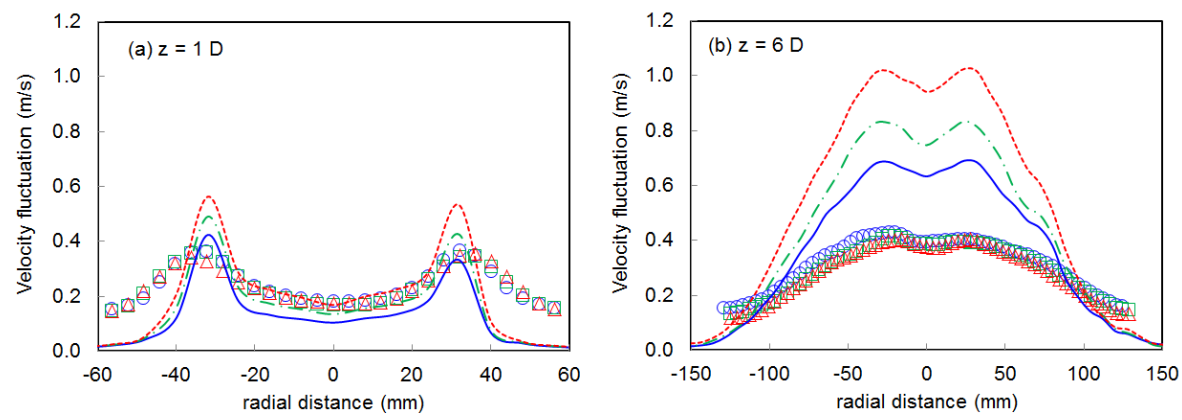

Figure 14: Predictions of the vertical velocity fluctuation (i.e., rms) radial profiles for cases 1 , 2 and 3 at (a) $z=1 D$ and (b) $z=6 D$. (see Fig. 12 for the legend)
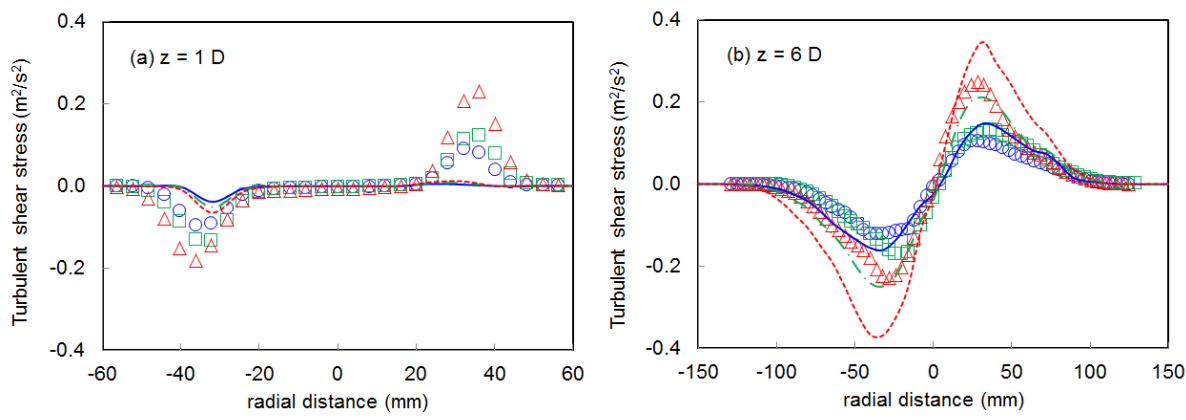

Figure 15: Predictions of the turbulent shear stress (i.e., Reynolds stress) radial profiles for cases 1, 2 and 3 at (a) $z=1 D$ and (b) $z=6 D$. (see Fig. 12 for the legend) 


\subsubsection{Parallel computing and computational times}

\subsubsection{Influence of the cell size and mesh resolution (case 1)}

Table 5 shows that refining the cell size to $2 \mathrm{~mm}$ did not yield any improvement in comparison to $4 \mathrm{~mm}$, except at $r=300 \mathrm{~mm}$. Furthermore, according to [5], as a general guideline for wall functions in LES, it is recommended that the first grid cell falls within the $\log$ layer. A value $y^{+}=30$ would be considered highly resolved. On the grounds of such recommendation, the flow field near the ceiling for the base case (with $4 \mathrm{~mm}$ cell size) can be considered as be well-resolved because $y^{+}<16$ at the level of the ceiling in the centreline. 


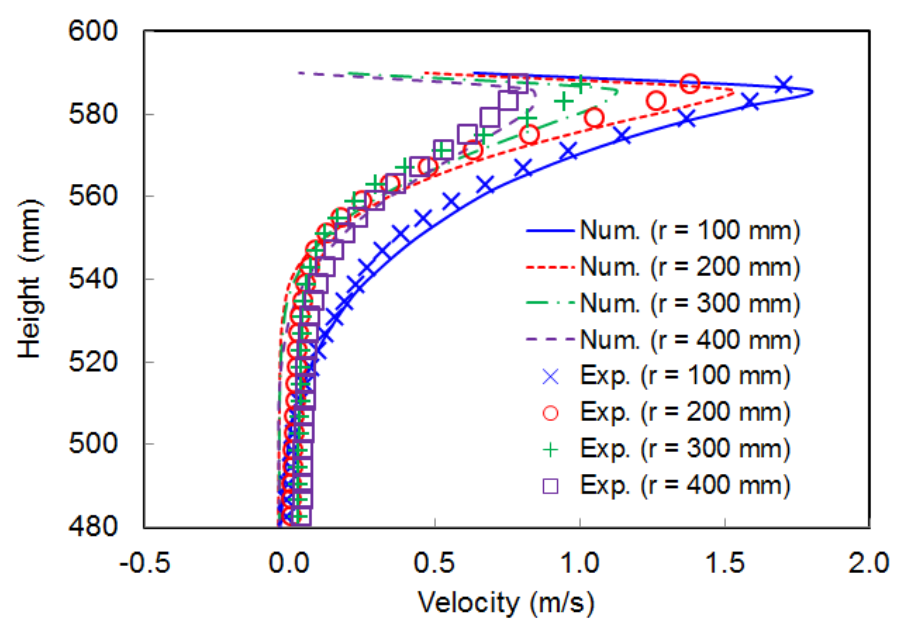

Figure 16: Experimental and numerical profiles of the ceiling jet velocity for case 1 at $r=100$ $\mathrm{mm}, r=200 \mathrm{~mm}, r=300 \mathrm{~mm}$ an $r=400 \mathrm{~mm}$.
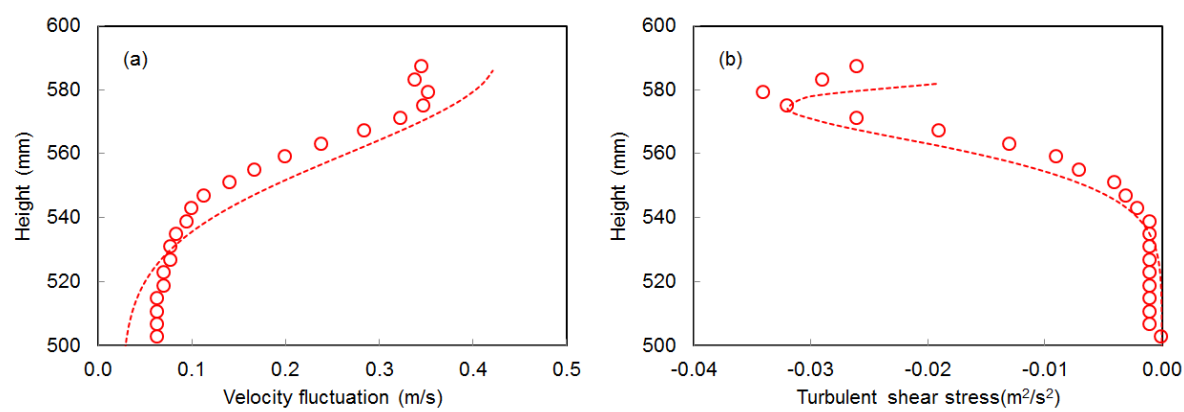

Figure 17: Experimental and numerical profiles of (a) the ceiling jet velocity fluctuation (i.e., rms) and (b) turbulent shear stress (i.e., Reynolds stress) for case 1 at $r=200 \mathrm{~mm}$.

\subsubsection{Influence of the domain size (case 1)}

The computational domain has been extended to $1280 \mathrm{~mm} \times 1280 \mathrm{~mm}$ in order to minimize the influence of the OPEN boundary condition on the flow field. Table 5 shows that extending the domain leads to minor changes in the ceiling jet velocities (with respect to the base case). Nevertheless, it is interesting to note that the deviations with the experimental data has been reduced by 1,2 and $3 \%$ at respectively $r=200 \mathrm{~mm}, r=300 \mathrm{~mm}$ and $r=400$ $\mathrm{mm}$, in comparison to the base case. This clearly shows how the measurement 
Table 5: Predicted $u_{\max }$ in the ceiling jet velocities (the numbers between parenthesis correspond to the relative deviation with the experimental data).

\begin{tabular}{|l|c|c|c|c|}
\hline & $r=100 \mathrm{~mm}$ & $r=200 \mathrm{~mm}$ & $r=300 \mathrm{~mm}$ & $r=400 \mathrm{~mm}$ \\
\hline Exp. data & 1.71 & 1.39 & 1.01 & 0.79 \\
\hline Base case & $1.78(+4 \%)$ & $1.52(+9 \%)$ & $1.12(+11 \%)$ & $0.84(+6 \%)$ \\
\hline$\Delta x=2 \mathrm{~mm}$ & $1.86(+9 \%)$ & $1.51(+9 \%)$ & $1.01(0 \%)$ & - \\
\hline Extended domain & $1.78(+4 \%)$ & $1.50(+8 \%)$ & $1.10(+9 \%)$ & $0.81(+3 \%)$ \\
\hline No-slip & $1.72(+1 \%)$ & $1.36(-2 \%)$ & $0.97(-4 \%)$ & $0.71(-10 \%)$ \\
\hline Modified Deardorff & $1.85(+8 \%)$ & $1.53(+11 \%)$ & $1.13(+12 \%)$ & $0.83(+5 \%)$ \\
\hline Adiabatic & $1.80(+6 \%)$ & $1.52(+9 \%)$ & $1.12(+11 \%)$ & $0.83(+5 \%)$ \\
\hline
\end{tabular}

points that are closer to the OPEN boundary of the computational domain are more affected by the numerical treatment of the latter.

\subsubsection{Influence of the wall function (case 1)}

According to the results displayed in Table 5, the no-slip BC provides an overall better agreement with the experimental data in $u_{\max }$ in comparison to the best case. Nevertheless, stronger discrepancies have been observed for the boundary layer width, $\delta_{u}$ (not shown here). Therefore, it is recommended to use the wall law.

\subsubsection{Influence of the turbulent viscosity model (case 1)}

According to Table 5, sufficiently away from the jet axis (i.e., at $r>200$ $\mathrm{mm}$ ), the results of $u_{\max }$ are altered by less than $2 \%$. The modified Deardorff model can be considered as a very good alternative to the dynamic Smagorinsky option, especially given the reduction in the computational time that it offers.

\subsubsection{Influence of the ceiling thermal boundary conditions (case 1)}

As shown in Table 5, the effect of the adiabatic wall $\mathrm{BC}$ is negligible. 


\subsubsection{Main results for the three cases}

The outcome of the base case model settings in displayed in Tables 6 and 7

for the three cases. The results are examined in Table 6 in terms of:

- maximum ceiling-jet velocity, $u_{\max }$,

- boundary layer width, $\delta_{u}$ (i.e., the distance between the ceiling and the location at which $u=u_{\max }$ ), and

- Gaussian momentum width, $\ell_{u}$ (i.e., the distance between the point where $u=u_{\max }$ and the point where $\left.u=e^{-1} u_{\max }\right)$.

These results show that the maximum ceiling jet velocities are overpredicted by 2 to $20 \%$. The predicted boundary layer thickness remained constant, with $\delta_{u}=4 \mathrm{~mm}$, except for two profiles (i.e., $r=400 \mathrm{~mm}$ in case 1 and $r=400$ $\mathrm{mm}$ in case 2). This is in accordance with the experimental data where the maximum ceiling jet velocity has been systematically recorded at $\delta_{u}=3 \mathrm{~mm}$. It is interesting to note that this experimental observation for $\delta_{u}$ (that is predicted numerically) is in contradiction with the reduced-scale experiments reported in [9. In [9], the ceiling jet boundary layer thickness has been observed to increase with increased distance from the vertical jet axis. The measurement distances $r / H$ are comparable. It must be noted however that the Froude number in 9] varied between 0.5 and 2.2, whereas for the cases at hand $F r$ varied between 3.9 and 6.3. The increased initial momentum herein could explain the difference. Regarding the third parameter, namely the Gaussian momentum width, the numerical predictions are within $20 \%$ of the experimental data. Note that the highest deviations ( $-17 \%$ for case 1 and $r=400 \mathrm{~mm}$ and $-18 \%$ for case 2 and $r=400 \mathrm{~mm}$ ) are mainly attributed to the overprediction of the boundary layer thickness by $5 \mathrm{~mm}$.

Table 7 displays the predicted non-dimensional ceiling-jet velocity fluctuations, $\left(\overline{u^{\prime} u^{\prime}}\right)^{1 / 2} / u_{\max }$, at the location of $u_{\max }$ (i.e., $z=H-\delta_{u}$ ) for the base case settings. The results show a very good agreement with the experimental 
Table 6: Predicted maximum ceiling-jet velocity, momentum Gaussian width and viscous boundary layer width. The numbers between parentheses represent either the relative deviation (in \%) or the absolute deviation with the experimental data.

\begin{tabular}{|c|c|c|c|c|}
\hline & $r(\mathrm{~mm})$ & $u_{\max }(\mathrm{m} / \mathrm{s})$ & $\ell_{u}(\mathrm{~mm})$ & $\delta_{u}(\mathrm{~mm})$ \\
\hline Case 1 & 100 & $1.78(+4 \%)$ & $26.2(+2 \%)$ & $4(+1 \mathrm{~mm})$ \\
\hline Case 1 & 200 & $1.52(+9 \%)$ & $19.5(+1 \%)$ & $4(+1 \mathrm{~mm})$ \\
\hline Case 1 & 300 & $1.12(+11 \%)$ & $22.3(+5 \%)$ & $4(+1 \mathrm{~mm})$ \\
\hline Case 1 & 400 & $0.84(+6 \%)$ & $23.9(-17 \%)$ & $8(+5 \mathrm{~mm})$ \\
\hline Case 2 & 100 & $2.14(+2 \%)$ & $25.9(+3 \%)$ & $4(+1 \mathrm{~mm})$ \\
\hline Case 2 & 200 & $1.81(+9 \%)$ & $19.6(+3 \%)$ & $4(+1 \mathrm{~mm})$ \\
\hline Case 2 & 300 & $1.34(+17 \%)$ & $21.8(-2 \%)$ & $4(+1 \mathrm{~mm})$ \\
\hline Case 2 & 400 & $1.00(+10 \%)$ & $25.3(-18 \%)$ & $8(+5 \mathrm{~mm})$ \\
\hline Case 3 & 100 & $2.62(+2 \%)$ & $25.2(+3 \%)$ & $4(+1 \mathrm{~mm})$ \\
\hline Case 3 & 200 & $2.22(+11 \%)$ & $20.0(+15 \%)$ & $4(+1 \mathrm{~mm})$ \\
\hline Case 3 & 300 & $1.65(+20 \%)$ & $21.8(+0 \%)$ & $4(+1 \mathrm{~mm})$ \\
\hline Case 3 & 400 & $1.26(+19 \%)$ & $28.4(-2 \%)$ & $4(+1 \mathrm{~mm})$ \\
\hline
\end{tabular}

Table 7: Predicted non-dimensional ceiling-jet velocity fluctuations, $\left(\overline{u^{\prime} u^{\prime}}\right)^{1 / 2} / u_{\max }$, at the location of $u_{\max }$ (i.e., $z=H-\delta_{u}$ ). The numbers between parentheses represent the absolute deviation with the experimental data.

\begin{tabular}{|c|c|c|c|c|}
\hline & $r=100 \mathrm{~mm}$ & $r=200 \mathrm{~mm}$ & $r=300 \mathrm{~mm}$ & $r=400 \mathrm{~mm}$ \\
\hline Case 1 & $28.1 \%(+7.5 \%)$ & $26.3 \%(+1.3 \%)$ & $30.2 \%(+0.1 \%)$ & $32.9 \%(+3.8 \%)$ \\
\hline Case 2 & $28.6 \%(+7.3 \%)$ & $25.9 \%(+0.9 \%)$ & $30.4 \%(-3.4 \%)$ & $31.9 \%(+0.3 \%)$ \\
\hline Case 3 & $28.3 \%(+5.0 \%)$ & $25.8 \%(+0.6 \%)$ & $29.6 \%(-0.1 \%)$ & $31.6 \%(-0.2 \%)$ \\
\hline
\end{tabular}

data, particularly sufficiently far from the impingement point (at $r>200 \mathrm{~mm}$ ) where the deviation is less than $4 \%$. 


\section{Conclusions}

The main objective of this paper (Part I) and the companion paper (Part II) 355 is to assess the current capabilities of Computational Fluid Dynamics (CFD) in the prediction of a two-phase flow in a configuration of interest to fire suppression. The scenario consists of a ceiling-mounted water mist nozzle positioned above a vertical jet of hot air that has been studied experimentally in 1]. The interaction of the water spray with the vertical jet is studied (among other aspects) in terms of penetration level of the water spray into the vertical jet of hot air.

Prior to two-phase flow simulations, it was essential to evaluate first in this paper (Part I) the capabilities of the CFD package (namely the Fire Dynamcis Simulator, FDS 6) in the prediction of the gas-phase using the Large Eddy Simulation (LES) approach. This is in line with the experimental program carried out in [1], where three campaigns were undertaken: $(i)$ impinging vertical jet on a horizontal ceiling plate, (ii) characterization of water spray only, and (iii) spray-jet interaction. We devoted this paper (Part I) to $(i)$ in order to be as assertive as possible as to the qualities of the gas-phase simulations before assessing the potential modelling difficulties that may be encountered in the simulation of the two-phase flows.

Through the simulations of the impinging vertical jet performed herein we examined the influence of several numerical settings. An extensive sensitivity study has shown that the dynamic Smagorinsky model for the turbulent viscosity provides the best agreement with the experimental data. The modified Deardorff model constitutes nevertheless a very good alternative option with a slightly lower level of agreement but significantly reduced computational times. The quality of the results for both cases depends on the cell size and the inlet modelling. The mesh sensitivity study demonstrates the suitability of a $4 \mathrm{~mm}$ cell size i.e., $D / \Delta x=64 / 4=16$. Another important modelling parameter studied here is the configuration of the inlet. It has been shown that it is necessary to model the inlet tube in order to avoid a spurious behaviour (a decrease in the 
vertical velocity) near the exit when the inlet air velocity is positioned flush to the computational domain. Furthermore, a calibration of the turbulence inflow to achieve a good agreement in the vertical velocity profiles. It is important to mention as well, that the quality of the simulations has not been assessed with respect to the mean flow field only. Second-moment profiles have also been checked with a relatively good overall agreement.

Based on the findings of this paper (Part I), we can proceed with confidence to the two-phase flow simulations in Part II.

\section{Acknowledgments}

Tarek Beji is a Postdoctoral Fellow of the Fund of Scientific ResearchFlanders (Belgium) (FWO Vlaanderen).

\section{References}

[1] X. Zhou, Characterization of interactions between hot air plumes and water sprays for sprinkler protection, Proceedings of the Combustion Institute 35 (2015) 2723-2729. doi:http://dx.doi.org/10.1016/j.proci.2014.05. 078 .

[2] K. Meredith, X. Zhou, S. Ebrahimzadeh, B. Merci, Numerical simulation of spray-plume interactions, 9th U.S. National Combustion Meeting.

[3] S. Ebrahimzadeh, G. Maragkos, T. Beji, B. Merci, Large eddy simulations of the ceiling jet induced by the impingement of a turbulent air plume, Fire Technology 52 (2016) 2093-2115. doi:https://doi.org/10.1007/ s10694-015-0561-3

[4] K. McGrattan, S. Hostikka, R. McDermott, J. Floyd, C. Weinschenk, K. Overholt, Fire dynamics simulator, technical reference guide, volume 
1: Mathematical model, National Institute of Standards and Technology,Gaithersburg, MD NIST Special Publication 1019 6th Edition (2013) $149 \mathrm{p}$.

[5] K. McGrattan, S. Hostikka, R. McDermott, J. Floyd, C. Weinschenk, K. Overholt, Fire dynamics simulator, user guide, National Institute of Standards and Technology, Gaithersburg, MD NIST Special Publication 1019 6th Edition (2013) 262 p.

[6] B. Toms, Large-eddy simulation of flow over a backward facing step: Assessment of inflow boundary conditions, eddy viscosity models and wall functions, Applied Mechanical Engineering 4. doi:http://dx.doi.org/ $10.4172 / 2168-9873.1000169$

[7] N. Jarrin, Synthetic inflow boundary conditions for the numerical simulation of turbulence, $\mathrm{PhD}$ thesis, The Univeristy of Manchester.

[8] R. Poletto, T. Craft, A. Revell, A new divergence free synthetic eddy method for the reproduction of inlet flow conditions for les, Flow, Turbulence and Combustion 91 (2013) 519-539. doi:https://doi.org/10.1007/ s10494-013-9488-2.

[9] V. Motevalli, C. Marks, Characterizing the unconfined ceiling jet under steady-state conditions: A reassessment, Proceedings of the International Association of Fire Safety Science (IAFSS) 3. doi:http://dx.doi.org/ 10.3801/iafss.fss.3-301. 